\title{
Metabolite Identification, Reaction Phenotyping, and Retrospective Drug-Drug Interaction Predictions of 17-Deacetylnorgestimate, the Active Component of the Oral Contraceptive Norgestimate ${ }^{\sqrt{\mathbb{S}}}$
}

\author{
Deepak Ahire, ${ }^{1}$ Sarmistha Sinha, ${ }^{1}$ Barry Brock, Ramaswamy lyer, Sandhya Mandlekar, \\ and Murali Subramanian \\ Pharmaceutical Candidate Optimization, Biocon Bristol-Myers Squibb R\&D Center, Syngene International Ltd., Bangalore, India \\ (D.A., S.S., M.S.); Pharmaceutical Candidate Optimization, Bristol-Myers Squibb, Pennington, New Jersy (B.B., R.I.); and \\ Pharmaceutical Candidate Optimization, Bristol-Myers Squibb India Ltd., Bangalore, India: (S.M.)
}

Received October 17, 2016; accepted March 6, 2017

\begin{abstract}
Ortho Tri-Cyclen, a two-drug cocktail comprised of ethinylestradiol and norgestimate (13-ethyl-17-acetoxy-18, 19-dinor-17 $\alpha$-pregn-4-en-20yn-3 oxime), is commonly prescribed to avert unwanted pregnancies in women of reproductive age. In vivo, norgestimate undergoes extensive and rapid deacetylation to produce 17-deacetylnorgestimate (NGMN), an active circulating metabolite that likely contributes significantly to norgestimate efficacy. Despite being of primary significance, the metabolism and reaction phenotyping of NGMN have not been previously reported. Hence, detailed biotransformation and reaction phenotyping studies of NGMN with recombinant cytochrome P450 (P450), recombinant uridine $5^{\prime}$-diphospho-glucuronosyltransferases, and human liver microsomes in the presence and absence of selective P450 inhibitors were conducted. It was found that CYP3A4 plays a key role in NGMN
\end{abstract}

metabolism with a fraction metabolized $\left(f_{\mathrm{m}}\right)$ of 0.57 . CYP2B 6 and to an even lesser extent CYP2C9 were also observed to catalyze NGMN metabolism. Using this CYP3A4 $f_{m}$ value, the predicted plasma concentration versus time area under the curve (AUC) change in NGMN using a basic/mechanistic static model was found to be within 1.3-fold of the reported NGMN AUC changes for four modulators of CYP3A4. In addition to NGMN, we have also elucidated the biotransformation of norgestrel (NG), a downstream norgestimate and NGMN metabolite, and found that CYP3A4 and UGT1A1 have a major contribution to the elimination of $N G$ with a combined $f_{m}$ value of 1 . The data presented in this paper will lead to better understanding and management of NGMNbased drug-drug interactions when norgestimate is coadministered with CYP3A4 modulators.

\section{Introduction}

Ortho Tri-Cyclen (OTC) is commonly prescribed to avert unwanted pregnancies in women of reproductive age, and is increasingly recommended as hormone replacement therapy to manage menopausal symptoms. It consists of $0.035 \mathrm{mg}$ of ethinylestradiol (EE), and $0.18,0.215$, or $0.25 \mathrm{mg}$ of norgestimate (Schwartz et al., 2009). It contains two hormones: 1) aprogestin (norgestimate), which suppresses ovulation, and 2) an estrogen (EE), which suppresses production of follicle-stimulating and luteinizing hormones (Becker, 1990; Corson, 1990; McGuire et al., 1990; Huber, 1991; Bringer, 1992; Kafrissen, 1992; Kaplan, 1995). Since OTC is prescribed to prevent pregnancy, maintaining efficacious levels of its components is crucial. However, this is complicated by the fact that the active components of OTC are very susceptible to drug-drug interactions (DDIs), with numerous DDIs having been reported thus far [see "Interactions between Antiretrovirals (ARVs) and Hormonal Contraceptives" (http://hivclinic. ca/main/drugs_interact_files/Oral\%20Contraceptive-int.pdf)]. These

${ }^{1}$ D.A. and S.S. contributed equally to this work.

https://doi.org/10.1124/dmd.116.073940.

S This article has supplemental material available at dmd.aspetjournals.org.
DDIs have usually been attributed to modulation of CYP3A4 activity (Hariparsad et al., 2004; Zhang et al., 2011).

Norgestimate (13-ethyl-17-acetoxy-18，19-dinor-17 $\alpha$-pregn-4-en20yn-3 oxime; norgestrel-3-oxime-17-acetate) is a synthetic steroid that possesses antiprogestational and antifertility activity (Becker, 1990; Corson, 1990; McGuire et al., 1990; Huber, 1991; Bringer, 1992; Kafrissen, 1992; Kaplan, 1995). Current understanding suggests that norgestimate is a prodrug and 17-deacetylnorgestimate (NGMN) is the active metabolite that likely exerts efficacy. Norgestimate and NGMN likely have equivalent potencies; however, NGMN has much higher systemic exposures, and hence likely contributes to contraceptive action much more than norgestimate (McGuire et al., 1990; Kafrissen, 1992). In the preclinical species of rats, dogs, and monkeys, orally administered ${ }^{14} \mathrm{C}$ norgestimate was rapidly absorbed with maximum radioactivity being detected in plasma within 4 hours of administration and with an elimination half-life of 30-67 hours. In women, ${ }^{14} \mathrm{C}$ norgestimate was also rapidly absorbed with maximal circulating radioactivity observed in 30 minutes to 2 hours and eliminated with a terminal half-life of 45-71 hours (Alton et al., 1984; McGuire et al., 1990; Kaplan, 1995; Schwartz et al., 2009). Upon oral administration of $0.18 \mathrm{mg}$ of norgestimate, the peak norgestimate serum concentrations were only $270 \mathrm{pM}$, whereas

ABBREVIATIONS: ATV, atazanavir; AUC, area under the curve; CYP3cide, 1-methyl-3-[1-methyl-5-(4-methylphenyl)-1H-pyrazol-4-yl]-4-[(3S)-3piperidin-1-ylpyrrolidin-1-yl]-1H-pyrazolo[3,4- $d$ ]pyrimidine; DDI, drug-drug interaction; EE, ethinylestradiol; EFZ, efavirenz; $f_{\mathrm{m}}$, fraction metabolized; HLM, human liver microsome; KTZ, ketoconazole; LC, liquid chromatography; LPR, lopinavir; MS, mass spectrometry; $m / z$, mass-to-charge; NG, norgestrel; NGMN, 17-deacetylnorgestimate; OTC, Ortho Tri-Cyclen; PI, protease inhibitor; P450, cytochrome P450; RTV, ritonavir; UDPGA, uridine 5'-diphosphoglucuronic acid; UGT, uridine 5'-diphospho-glucuronosyltransferase. 
1 hour after dosing NGMN concentrations were greater than $12 \mathrm{nM}$, and high concentrations were seen even 36 hours after dosing (Alton et al., 1984; McGuire et al., 1990; Kaplan, 1995; Schwartz et al., 2009). Hence, the long circulating radioactivity half-life likely stems from NGMN and not norgestimate.

In vitro, it was found that norgestimate is rapidly decaetylated in multiple matrices to produce NGMN which is subsequently metabolized to produce norgestrel (NG), which undergoes further metabolism (Madden and Back, 1991; Wild et al., 1991, 1993). In human liver microsome (HLM) preps, NGMN was formed in the absence of NADPH from norgestimate, whereas in the presence of NADPH, NGMN, 3-keto norgestimate, NG, and other metabolites were formed. Normal colon samples, human endometrial cancer cell lines (HEC-1A), endometrial tissue, stomach tissue, and normal and malignant breast cells in culture were also found to convert norgestimate to NGMN (Madden and Back, 1991; Wild et al., 1993). As such, cytochrome P450 (P450) expression is not expected in these cells, suggesting that a wide variety of hydrolytic enzymes are capable of converting norgestimate to NGMN. The fact that norgestimate metabolism is fundamentally catalyzed by enzymes other than P450s, and in multiple tissues and organs, makes norgestimate resistant to DDIs, since such DDIs are typically mediated through P450s. In contrast, NGMN metabolism was found to be NADPH dependent, and a significant correlation was observed between NGMN metabolism and P450 content (Madden and Back, 1991). To summarize, norgestimate is metabolized rapidly to an active metabolite, NGMN, which circulates at efficacious concentrations with a long elimination half-life and likely contributes significantly to efficacy. Currently, there is no understanding of the enzyme families responsible for the clearance of NGMN and NG. Hence, to predict and enable a retrospective understanding of NGMN and NG DDIs, metabolite identification and reaction phenotyping studies were undertaken to determine P450 and uridine 5' -diphospho-glucuronosyltransferase (UGT) isoforms involved in NGMN and NG metabolism. NGMN DDIs have been reported when lopinavir (LPR)/ritonavir (RTV), RTV/atazanavir (ATV), or fold of plasma efavirenz (EFZ) were coadministered with norgestimate; the actual concentration versus time area under the curve (AUC) changes were compared with retrospectively calculated AUC changes based on the determined fraction metabolized $\left(f_{\mathrm{m}}\right)$ value of CYP3A4 (Vogler et al., 2010; Sevinsky et al., 2011; Zhang et al., 2011). Our data and analyses from these studies would lead to more effective management of clinical trials and marketed drug use in women of child bearing potential who have been prescribed norgestimate.

\section{Materials and Methods}

\section{Chemicals}

Norgestimate $\left(\mathrm{C}_{23} \mathrm{H}_{31} \mathrm{NO}_{3}\right)$, norethindrone $\left(\mathrm{C}_{20} \mathrm{H}_{26} \mathrm{O}_{2}\right)$, napthoflavone $\left(\mathrm{C}_{19} \mathrm{H}_{12} \mathrm{O}_{2}\right)$, thio TEPA $\left(\mathrm{C}_{6} \mathrm{H}_{12} \mathrm{~N}_{3} \mathrm{PS}\right)$, quercetin $\left(\mathrm{C}_{15} \mathrm{H}_{10} \mathrm{O}_{7}\right)$, sulphaphenazole $\left(\mathrm{C}_{15} \mathrm{H}_{14} \mathrm{~N}_{4} \mathrm{O}_{2} \mathrm{~S}\right)$, benzylnirvanol $\left(\mathrm{C}_{18} \mathrm{H}_{18} \mathrm{~N}_{2} \mathrm{O}_{2}\right)$, quinidine $\left(\mathrm{C}_{20} \mathrm{H}_{24} \mathrm{~N}_{2} \mathrm{O}\right)$, glutathione $\left(\mathrm{C}_{10} \mathrm{H}_{17} \mathrm{~N}_{3} \mathrm{O}_{6} \mathrm{~S}\right)$, CYP3cide $\left(\mathrm{C}_{26} \mathrm{H}_{32} \mathrm{~N}_{8}\right.$; 1-methyl-3-[1-methyl-5-(4-methylphenyl)$1 H$-pyrazol-4-yl]-4-[(3S)-3-piperidin-1-ylpyrrolidin-1-yl]- $1 H$-pyrazolo[3,4- $d]$ pyrimidine), ketoconazole [(KTZ), $\left.\mathrm{C}_{26} \mathrm{H}_{28} \mathrm{Cl}_{2} \mathrm{~N}_{4} \mathrm{O}_{4}\right]$, ATV $\left(\mathrm{C}_{38} \mathrm{H}_{52} \mathrm{~N}_{6} \mathrm{O}_{7}\right)$, NGMN $\left(\mathrm{C}_{21} \mathrm{H}_{29} \mathrm{NO}_{2}\right)$, and $\mathrm{NG}\left(\mathrm{C}_{21} \mathrm{H}_{28} \mathrm{O}_{2}\right)$ were purchased from Sigma Aldrich (St. Louis, MO). High-performance liquid chromatography (LC) grade acetonitrile (ACN, $\left.\mathrm{C}_{2} \mathrm{H}_{3} \mathrm{~N}\right)$, methanol $\left(\mathrm{CH}_{4} \mathrm{O}\right)$, formic acid $\left(\mathrm{H}_{2} \mathrm{CO}_{2}\right)$, potassium dihydrogen phosphate $\left(\mathrm{KH}_{2} \mathrm{PO}_{4}\right)$, and dipotassium hydrogen phosphate $\left(\mathrm{K}_{2} \mathrm{HPO}_{4}\right)$ were purchased from Merck Specialties Private Limited (Mumbai, India). NADPH $\left(\mathrm{C}_{21} \mathrm{H}_{29} \mathrm{~N}_{7} \mathrm{O}_{17} \mathrm{P}_{3}\right)$ was purchased from (Sisco Research Laboratory Ltd., Mumbai, India) and five mixed-gender pooled HLMs, recombinant UGTs, and recombinant P450s were purchased from Corning (Corning, NY).

\section{Instruments}

The LC-mass spectrometry (MS) systems used in the study were the following: 1) LC-MS Orbitrap (Thermo Scientific, Bremen, Germany), equipped with an Agilent (Santa Clara, CA) 1200 high-performance LC system for in vitro incubations in HLMs, and 2) QTRAP 5500 (AB Sciex, Concord, Ontario, Canada), equipped with a Waters (Milford, MA) ACQUITY ultra-performance LC system for reaction phenotyping.

In Vitro Incubations in HLMs for Metabolite Profiling of NGMN and NG. A typical P450 microsomal assay was carried out in HLMs with NGMN and NG, wherein both NGMN and NG $(30 \mu \mathrm{M})$ were separately preincubated with HLMs $(1 \mathrm{mg} / \mathrm{ml})$ in phosphate buffer $(0.1 \mathrm{M})$ at $\mathrm{pH} 7.4$ for 5 minutes. Reactions were initiated by addition of NADPH $(1 \mathrm{mM})$ and incubated for 60 minutes at $37^{\circ} \mathrm{C}$ in a total reaction volume of $1000 \mu \mathrm{l}$. Control incubations were performed in the absence of NADPH. At 0 and 60 minutes, $300 \mu \mathrm{l}$ aliquots were taken and quenched with an equal volume of ACN precipitate the proteins. Then, the reaction mixture was vortexed, centrifuged at $14000 \mathrm{~g}$ for 10 minutes, and the resulting supernatants were analyzed in the LTQ-Velos Orbitrap.

For determination of NADPH and UGT-mediated metabolism, both NGMN and NG $(30 \mu \mathrm{M})$ were preincubated in Tris- $\mathrm{HCl}$ buffer $(0.1 \mathrm{M})$ at $\mathrm{pH} 7.4$ containing $10 \mathrm{mM} \mathrm{MgCl}$ and $1 \mathrm{mg} / \mathrm{ml}$ alamethacin-treated HLMs. The HLMs were pretreated for 20 minutes with alamethacin $(50 \mu \mathrm{g} / \mathrm{mg}$ protein) on ice. The reaction was initiated by the addition of uridine $5^{\prime}$-diphosphoglucuronic acid [(UDPGA), $3 \mathrm{mM}$ ] and NADPH (1 mM). The incubations and sample processing were carried out in a similar fashion as described previously, and samples were analyzed by LTQ-Velos Orbitrap. Control experiments were performed in the absence of NADPH and UDPGA.

Reaction Phenotyping. In a typical assay, both NGMN and NG $(1 \mu \mathrm{M})$ were preincubated for 5 minutes with recombinant CYP3A4, CYP3A5, CYP2C9, CYP2C8, CYP2C19, CYP2D6, CYP1A2, CYP2E1, CYP2A6, and CYP2B6 $(25 \mathrm{nM})$ in 96 -well plates containing phosphate buffer $(0.1 \mathrm{M})$ at $\mathrm{pH}$ 7.4. The reaction was initiated by addition of NADPH $(1 \mathrm{mM})$ and incubated for 60 minutes at $37^{\circ} \mathrm{C}$. Aliquots were taken at $0,3,15$, and 60 minutes, and quenched with two volumes of acetonitrile containing norethindrone as an internal standard. Sample processing was carried out in a similar fashion as described in the previous section and analyzed by ultra-performance LC-MS/MS. Reaction phenotyping of NGMN was also performed with HLMs in the presence of specific inhibitors. The selective P450 inhibitors were alpha-napthoflavone (CYP1A1 inhibitor at $1 \mu \mathrm{M})$, thio-TEPA (CYP2B6, $50 \mu \mathrm{M})$, quercetin (CYP2C8, $20 \mu \mathrm{M})$, sulphaphenazole (CYP2C9, $10 \mu \mathrm{M})$, benzylnirvanol (CYP2C19, $1 \mu \mathrm{M})$, quinidine (CYP2D6, $1 \mu \mathrm{M}$ ), CYP3cide (CYP3A4, $1 \mu \mathrm{M}$ ), and KTZ (CYP3A4 and CYP3A5, $1 \mu \mathrm{M})$. Thio-TEPA and CYP3cide are mechanism-based inhibitors; hence, both those inhibitors were preincubated with the HLM and NAPDH mixture for 15 minutes and reaction was initiated by addition of NGMN $(1 \mu \mathrm{M})$. For the other inhibitors, a mixture of NGMN $(1 \mu \mathrm{M})$, HLMs $(1 \mathrm{mg} / \mathrm{ml})$, and inhibitors were preincubated for 5 minutes and reaction was initiated by addition of NADPH $(1 \mathrm{mM})$. The reaction was incubated for 60 minutes at $37^{\circ} \mathrm{C}$ and aliquots were taken at 0,30 , and 60 minutes, quenched with two volumes of $\mathrm{ACN}$ containing norethindrone as an internal standard, and the samples were processed for analysis as described previously. To determine UGT activity, NGMN and NG $(1 \mu \mathrm{M})$ were preincubated with rUGT1A1, rUGT1A4, rUGT1A6, rUGT2B4, and rUGT2B7 $(1 \mathrm{mg} / \mathrm{ml})$ in 96-well plates, with alamethacin pretreatment, as described in the previous section. The reaction was initiated by addition of UDPGA $(5 \mathrm{mM})$ followed by an incubation of 45 minutes at $37^{\circ} \mathrm{C}$. Aliquots were taken at $0,5,15,30$, and 45 minutes. Additionally, in a separate incubation NG $(1 \mu \mathrm{M})$ was incubated with rUGT1A1 $(1 \mathrm{mg} / \mathrm{ml})$ and UDPGA $(5 \mathrm{mM})$ for 5 minutes with aliquots taken at $0,1,2,3,4$, and 5 minutes. Samples were processed and analyzed as previously described in this section. In another set of incubations, NG $(1 \mu \mathrm{M})$ was incubated with alamethacin-treated HLMs $(1 \mathrm{mg} / \mathrm{ml})$ as previously described, with and without KTZ (CYP3A4 and CYP3A5 inhibitor at $1 \mu \mathrm{M}$ ) and ATV (UGT1A1 and CYP3A4/3A5 inhibitor at $1 \mu \mathrm{M}$ ). The reaction was initiated by addition of NADPH $(1 \mathrm{mM})$ and UDPGA $(5 \mathrm{mM})$. The incubation was carried out at $37^{\circ} \mathrm{C}$ for 45 minutes, aliquots were taken at $0,5,15,30$, and 45 minutes, and samples were processed as previously described.

Determining the $f_{\mathbf{m}}$ Value. The $f_{\mathrm{m}}$ values for NGMN were determined from their incubation in the presence and absence of specific inhibitors in HLMs, whereas the $f_{\mathrm{m}}$ values for NG were determined from separate incubations of NG with and without KTZ and ATV, as described in the previous section. The initial rate of disappearance $\left(k_{\mathrm{el}}\right)$ of NGMN and NG in the presence and absence of inhibitors was determined by plotting the natural log of $\%$ disappearance versus time; $f_{\mathrm{m}}$ was calculated using eq. 1 , as described in Yang et al. (2016). Sufficient turnover to calculate an elimination rate constant and half-life value was assessed by determining if the elimination slope was statistically different from zero 


$$
\begin{aligned}
& \% \text { inhibition }=100 \times \frac{k_{\mathrm{el}, \mathrm{no} \mathrm{inh}}-k_{\mathrm{el}, \text { with inh }}}{k_{\mathrm{el}, \text { no inh }}} \\
& f_{m}=\frac{\% \text { inhibition }}{\text { sum of total } \% \text { inhibition across P450 isoforms }}
\end{aligned}
$$

Prediction of NGMN DDIs. Based on a survey of the literature, significant NGMN (as a victim) DDIs were observed in three studies, where LPR/RTV, RTV/ATV, or EFZ were coadministered with norgestimate (Vogler et al., 2010; Sevinsky et al., 2011; Zhang et al., 2011). Other studies also reported victim DDIs of NGMN but had insufficient data to facilitate retrospective calculations. The University of Washington (Seattle, WA) DDI database was queried for induction and reversible and irreversible inhibition of LPR, RTV, ATV, and EFZ and the values are summarized in Table 1. NGMN victim DDIs were predicted using eq. 2 from the Food and Drug Administration guidance document with the incorporation of the $f_{\mathrm{m}}$ value of CYP3A4 determined from our studies (U.S. Department of Health and Human Services, 2012)

$$
\frac{\mathrm{AUC}_{\mathrm{I}}}{\mathrm{AUC}}=\frac{1}{\left(A_{\mathrm{h}} \times B_{\mathrm{h}} \times C_{\mathrm{h}}\right) \times f_{\mathrm{m}}+\left(1-f_{\mathrm{m}}\right)}
$$

where $\mathrm{AUC}_{\mathrm{i}} / \mathrm{AUC}$ is the predicted ratio of NGMN of the plasma concentration versus time AUC in the presence and absence of the inhibitor; and $f_{\mathrm{m}}$ is the value of NGMN metabolized by the affected P450. Only the hepatic component of inhibition has been considered since $F_{\mathrm{g}}$ (intestinal availability) data, a key parameter governing the extent of inhibition, for the inhibitors are not available

The terms for reversible inhibition, irreversible inhibition, and induction of the perpetrators are $A_{\mathrm{h}}, B_{\mathrm{h}}$ and $C_{\mathrm{h}}$, respectively, and are defined as follows in eqs. 3, 4, and 5:

$$
A=\frac{1}{1+\left(\left[I_{\mathrm{h}}\right] / K_{\mathrm{i}}\right)}
$$

where $\left[I_{\mathrm{h}}\right.$ is the in vivo inhibitor concentration of the perpetrator and $K_{\mathrm{i}}$ is the perpetrator reversible inhibitor constant

$$
B=\frac{1}{1+\left\{\left([I]_{\mathrm{h}} \times k_{\text {inact }}\right) /\left[k_{\operatorname{deg}} \times\left(K_{\mathrm{I}}+[I]_{\mathrm{h}}\right)\right]\right\}}
$$

where $K_{\mathrm{I}}$ is the perpetrator irreversible inactivator inhibition constant; $k_{\text {inact }}$ is the maximum inactivation rate constant of the perpetrator; and $k_{\mathrm{deg}}$ is the in vivo firstorder degradation rate constant of the affected $\mathrm{P} 450$

$$
C=1+\frac{d \times E_{\max } \times[I]_{\mathrm{h}}}{[I]_{\mathrm{h}}+\mathrm{EC}_{50}}
$$

where $E_{\max }$ is the fold induction in enzyme activities of the perpetrator; and $\mathrm{EC}_{50}$ is the perpetrator concentration of the inducer causing half-maximal induction. $\mathrm{d}$ was assumed to be 1 as advised in the Food and Drug Administration guidance (U.S. Department of Health and Human Services, 2012).
Three values of []$_{\mathrm{h}}$ were used in the equation: total maximal plasma concentration $\left(C_{\max }\right)$, unbound $C_{\max }\left(f_{\mathrm{u}} \times C_{\max }\right)$, and the unbound portal vein concentration from eq. 6 (U.S. Department of Health and Human Services, 2012):

$$
[I]_{\mathrm{h}}=f_{\mathrm{u}} \times[I]_{\max }+\frac{F_{\mathrm{a}} \times K_{\mathrm{a}} \times \text { dose }}{Q_{\mathrm{h}}}
$$

where $f_{\mathrm{u}}$ is the fraction of the inhibitor unbound in the blood of the perpetrator; $I_{\max }$ is the $C_{\max }$ value of the perpetrator; $F_{\mathrm{a}}$ is the fraction absorbed after oral administration of the perpetrator; $K_{\mathrm{a}}$ is the first-order absorption rate constant of the perpetrator; and $Q_{\mathrm{h}}$ is the hepatic blood flow. For the purposes of these calculations, the following values were assumed: $k_{\mathrm{deg}}=0.000321$ minute $^{-1}, F_{\mathrm{a}}=1$, $K_{\mathrm{a}}=0.1$ minute $^{-1}$, and $Q_{\mathrm{h}}=1.5 \mathrm{l} / \mathrm{min}$. These are the conservative values for DDI predictions as mentioned in U.S. Department of Health and Human Services (2012). The $f_{\mathrm{u}}$ values for LPR, RTV, ATV, and EFZ were obtained from the literature as $0.008,0.0027,0.135$, and 0.006 , respectively (Almond et al., 2005; Zhang et al., 2005; Kalvass et al., 2007; Aweeka et al., 2010; Delille et al., 2014).

\section{Results}

Metabolite Profile of NGMN in HLMs. A typical LC-MS chromatogram of NGMN metabolites produced in NADPH- and UDPGA-supplemented HLMs is shown in Fig. 1, where NGMN eluted at 28.31 minutes. In addition to NGMN, seven metabolites including NG (denoted as Met7) and other oxidative metabolites (denoted as Met1, Met2, Met3, Met4, Met5, and Met6) were detected. These metabolites eluted at 15.77 minutes (Met1), 16.55 minutes (Met2), 19.49 minutes (Met3), 21.35 minutes (Met4), 24.14 minutes (Met5), 26.47 minutes (Met6), and 27.02 minutes (Met7) (Fig. 1; Supplemental Fig. 2). The relative abundance of parent and metabolites is shown in Table 2; the predominant metabolic pathway was P450-mediated oxidation and hydrolysis of oxime moiety into ketone to produce NG. Trace amounts of a glucuronide were detected by MS only.

The structures of metabolites of NGMN were elucidated based on exact mass and product ion mass spectral analysis (Supplemental Fig. 2). NGMN displayed a molecular ion peak of 328.2271. The product ion spectra of NGMN exhibited characteristic daughter ion peaks at massto-charge $(\mathrm{m} / \mathrm{z})$ ratios of $310.2165,292.206,260.2009,264.1747$, 124.0754, and 282.1852 (Fig. 2). The product ions of $\mathrm{m} / \mathrm{z} 310.2165$ and 292.2060 were produced by water loss. The ion of $\mathrm{m} / \mathrm{z} 260.2009$ is a result of $\mathrm{C}-\mathrm{C}$ bond cleavage and simultaneous removal of but-3-yn-2-ol from the cyclopentane ring. The ions of $\mathrm{m} / \mathrm{z} 264.1747$ and 282.1852 were

\begin{tabular}{|c|c|c|c|c|c|c|c|c|c|}
\hline \multirow{2}{*}{ Drug } & \multirow{2}{*}{ Dose } & \multicolumn{3}{|c|}{ CYP3A4 } & \multicolumn{2}{|c|}{ CYP3A4 } & \multicolumn{2}{|c|}{ NGMN } & \multirow{2}{*}{ Reference } \\
\hline & & $K_{\mathrm{I}}$ & $k_{\text {inact }}$ & $K_{\mathrm{i}}$ & $\mathrm{EC}_{50}$ & $E_{\max }$ & $\mathrm{AUC}_{\mathrm{i}}$ & AUC & \\
\hline & $m g$ & $n M$ & $\min ^{-1}$ & $n M$ & $\mu M$ & & $p g \cdot h / m l$ & $\mathrm{pg} \cdot \mathrm{h} / \mathrm{ml}$ & \\
\hline LPR & 400 & 1000 & 0.11 & 7300 & & & 138 & 76 & $\begin{array}{l}\text { Weemhoff et al. (2003); Ernest et al. (2005); Fahmi et al. } \\
\text { (2008); Vogler et al. (2010) }\end{array}$ \\
\hline RTV study 1 & 100 & 170 & 0.4 & 40 & 1000 & 68.5 & 138 & 76 & $\begin{array}{l}\text { Weemhoff et al. (2003); Ernest et al. (2005); Fahmi et al. } \\
\text { (2008); Vogler et al. (2010) }\end{array}$ \\
\hline ATV & 300 & 50 & 0.049 & 2300 & & & 35,022 & 19,188 & $\begin{array}{l}\text { Ernest et al. (2005); Fahmi et al. (2008); Hyland et al. (2008); } \\
\text { Zhang et al. (2011) }\end{array}$ \\
\hline RTV study 2 & 100 & 170 & 0.4 & 40 & 1000 & 68.5 & 35,022 & 19,188 & $\begin{array}{l}\text { Ernest et al. (2005); Fahmi et al. (2008); Hyland et al. (2008); } \\
\text { Zhang et al. (2011) }\end{array}$ \\
\hline EFZ & 600 & & & 40,000 & 3900 & 6.5 & 6522 & 18,328 & $\begin{array}{l}\text { Sevinsky et al. (2011); Siccardi et al. (2013); Xu and Desta } \\
\text { (2013) }\end{array}$ \\
\hline
\end{tabular}
produced by removal of the ethyl moiety and by water loss. The ion 124.0754 resulted from cleavage of the $\mathrm{C}-\mathrm{C}$ bond and removal of methyloctahydro- $1 H$-inden-1-ol, which corresponded to 5-hydroxyimino

TABLE 1

In vitro values of perpetrators and NGMN AUC values from clinical studies

$K_{\mathrm{I}}$, perpetrator irreversible inhibition constant; $k_{\text {inact }}$, perpetrator maximum irreversible inactivation rate constant; $K_{\mathrm{i}}$, perpetrator reversible inhibitor constant; $\mathrm{EC}_{50}$, perpetrator concentration of

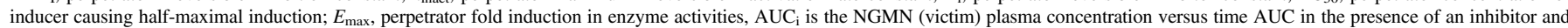
AUC is the NGMN plasma concentration versus time AUC in the absence of an inhibitor. 

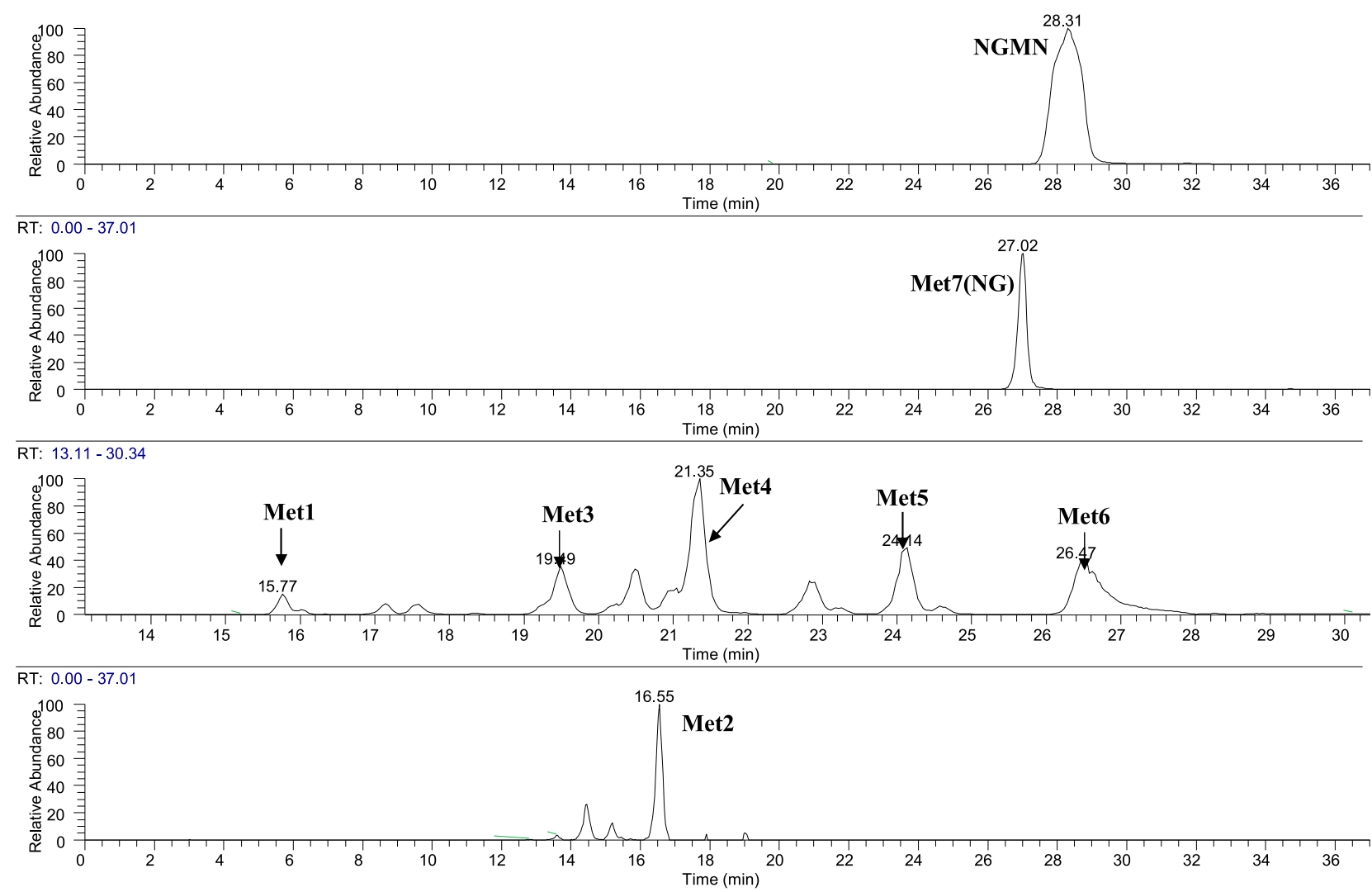

Fig. 1. LC-MS chromatogram of NGMN and its metabolites after incubating the substrates in NADPH-supplemented HLMs. Separations were achieved on a Thermo Hypersil Gold C18 column and detected by a LTQ-Velos Orbitrap. The different panels contain the extracted ion chromatograms of parent and various metabolites.

cyclohex-3-enyl methylium ion. Oxidative metabolites denoted as Met1 and Met3-6 exhibited a molecular ion peak at $\mathrm{m} / \mathrm{z}$ 344.222, an addition of $15.9949 \mathrm{Da}$ to the molecular ion of NGMN at $\mathrm{m} / \mathrm{z}$ 328.2271, suggesting mono-hydroxylation of parent. The presence of a fragment ion of $\mathrm{m} / \mathrm{z} 124.0754$ as unaltered and addition of $15.9949 \mathrm{Da}$ to the fragment ions of $m / z$ 310.2165, 264.1747, and 124.0754 in Met1 suggested that oxidation took place on the decahydronaphthalene ring. The molecular ion peak of Met2 was 360.2169; an addition of 31.9898 Da to the molecular ion of NGMN at $m / z 328.2271$, suggesting di-hydroxylation of parent. The collision-induced dissociation spectra of Met2 exhibited ions at $\mathrm{m} / \mathrm{z}$ 342.2064, 324.1958, 280.1696, 124.0757 , and 298.1802, suggesting addition of $31.9898 \mathrm{Da}$ to the fragment ions of $m / z, 310.2165$ and 292.2060 and addition of 15.9949 Da to the fragment ions of $\mathrm{m} / \mathrm{z}, 264.1747$ and 282.1852; however the fragment ion of $\mathrm{m} / \mathrm{z} 124.0754$ remained, which implied that di-hydroxylation had occurred on both the decahydrophenanthren ring and ethyl moiety. The product ion spectra of Met3 exhibited modification of $\mathrm{m} / \mathrm{z} 310.216,292.2060$, and 260.2009 fragment ions by addition of 15.9949 Da; however, the fragment ion of $\mathrm{m} / \mathrm{z}$ 124.0754 remained unaltered, suggesting hydroxylation had occurred on the ethyldecahydronaphthalene ring. The collision-induced dissociation spectra of Met 4 revealed addition of $15.9949 \mathrm{Da}$ to the fragment ions of $\mathrm{m} / \mathrm{z} 310.2465,292.2060$, and 260.2009, whereas the fragment ions of $\mathrm{m} / \mathrm{z}, 124.0754$ and 282.1852 remained unaltered, which indicated that oxidation had occurred on the ethyl moiety. The presence of unaltered fragmentations of $\mathrm{m} / \mathrm{z}, 260.2009$ and 124.0754 and the addition of 15.9949 Da to the fragment ion of $\mathrm{m} / \mathrm{z}$ 292.2060 in Met5 suggested that oxidation had occurred on the cyclopentane ring. The product ion spectra of Met6 exhibited ions at $\mathrm{m} / \mathrm{z} 326.2115$, $308.2009,276.1958$, and 280.1696, suggesting addition of 15.9949 Da to the fragment ions of $\mathrm{m} / \mathrm{z} 310.2165,292.2060,260.2009$, and 264.1747, which indicated oxidation had occurred on the decahydrophenanthren ring.

Metabolite Profile of NG in HLMs. In HLMs supplemented by NADPH and UDPGA six hydroxylated metabolites (denoted as Met8 and Met10-14) and one glucuronide metabolite of NG (assigned as Met9) were detected as the most abundant metabolites by both UV and MS (Fig. 3). The retention times of NG and its metabolites were 27.04 minutes (NG), 17.94 minutes (Met8), 18.63 minutes (Met9), 20.49 minutes (Met10), 20.74 minutes (Met11), 21.16 minutes (Met12), 22.06 minutes (Met13), and 22.36 minutes (Met14). The relative abundances of parent and metabolites are shown in Table 3. The predominant metabolic pathways of NG were found to be NADPHdependent oxidation and glucuronidation. The structures of metabolites of NG were elucidated based on exact mass and product ion mass

TABLE 2

Metabolite profile of NGMN after incubation of $30 \mu \mathrm{M}$ NGMN in $1 \mathrm{mM}$ NADPHsupplemented $1 \mathrm{mg} / \mathrm{ml}$ HLMs

\begin{tabular}{lclc}
\hline Parent/Metabolite & $\mathrm{RT}$ & $\mathrm{MH}^{+}$ & Relative Abundance (UV) \\
\hline & & & $\%$ \\
NGMN & 28.31 & 328.2271 & 83.0 \\
Met1 & 15.77 & 344.222 & 1.5 \\
Met2 & 16.55 & 360.2169 & 2.0 \\
Met3 & 19.49 & 344.222 & 2.0 \\
Met4 & 21.35 & 344.222 & 3.0 \\
Met5 & 24.14 & 344.222 & 2.0 \\
Met6 & 26.47 & 344.222 & 1.5 \\
Met7/NG & 27.02 & 313.2162 & 5.0 \\
\hline
\end{tabular}

$\mathrm{MH}^{+}$, Positive ion mass; RT, Retention Time. 


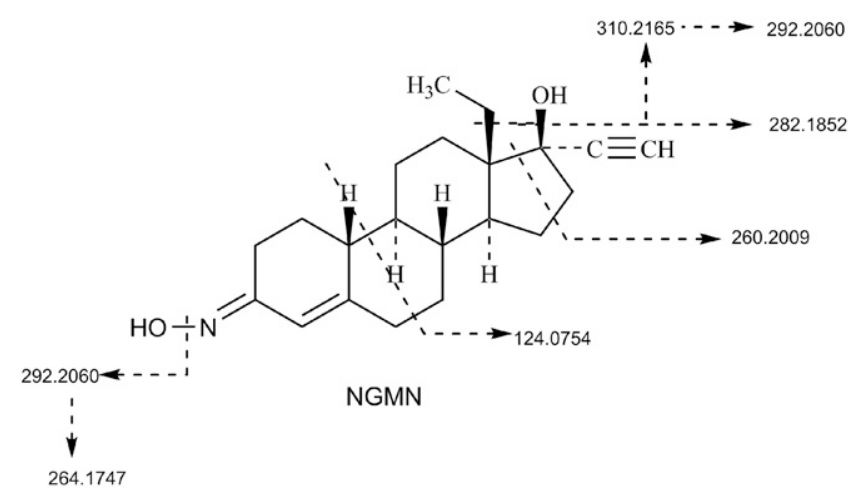

Fig. 2. Fragmentation of NGMN after its fragmentation in an LTQ-Velos-Orbitrap using collision-induced disassociation fragmentation.

spectral analysis (Supplemental Fig. 3). NG displayed a molecular ion peak of 313.2163. The product ion spectra of NG exhibited characteristic daughter ion peaks at $\mathrm{m} / \mathrm{z} 225.205,277.1951,267.1743,245.19$, 237.1638, and 109.0648 (Fig. 4). The product ions of $\mathrm{m} / \mathrm{z} 295.2050$ and 277.1951 were produced by water loss. The daughter ions of $\mathrm{m} / \mathrm{z}$ 267.1743 and 245.1900 were produced by removal of ethyl moiety, and simultaneous water loss from the cyclopentane ring, and by cleavage of a $\mathrm{C}-\mathrm{C}$ bond, and simultaneous removal of but-3-yn-2-ol from the cyclopentane ring, respectively. The product ion of $\mathrm{m} / \mathrm{z} 237.1638$ was formed by removal of prop-2-yn-1-ol from the cyclopentane ring and further water loss. The daughter ion of $\mathrm{m} / \mathrm{z}, 109.0648$ was produced from cleavage of a $\mathrm{C}-\mathrm{C}$ bond and removal of methyloctahydro- $1 H$-inden-1-ol, which corresponded to the 5-oxocyclohex-3-enyl methylene ion. Oxidative metabolites denoted as Met8 and Met10-14 exhibited a molecular ion peak of $\mathrm{m} / \mathrm{z} 329.2111$, an addition of 15.9949 Da to the molecular ion of NG at $\mathrm{m} / \mathrm{z}$ 313.2162, suggesting mono-hydroxylation of the parent. The collision ion dissociation spectra of Met8, Met10, and Met12 exhibited ions of $\mathrm{m} / \mathrm{z}, 293.19$ and 243.1743, suggesting the addition of 15.9949 Da followed by water loss to the fragment ions of $\mathrm{m} /$ $z$ 295.2050 and 245.1900, while the fragment ion of $\mathrm{m} / \mathrm{z} 109.0648$ remained unaltered, which implies that oxidation occurred on the ethyldecahydronaphthalene ring in the cases of Met8, Met10, and Met12. The collision-induced dissociation spectra of Met11 revealed addition of 15.9949 Da to the fragment ions of $\mathrm{m} / \mathrm{z}, 295.2050$ and 277.1951 and addition of $15.9949 \mathrm{Da}$ followed by water loss to fragment ion of $\mathrm{m} / \mathrm{z}$ 245.1900, whereas fragment ions of $m / z, 267.1743$ and 109.0648 remained unaltered, which indicated that oxidation had occurred on the ethyl moiety. The presence of fragment ions of $\mathrm{m} / \mathrm{z} 245.1900$ and 109.0648 as unaltered and addition of $15.9949 \mathrm{Da}$ to the fragment ion of $\mathrm{m} / \mathrm{z} 295.2050$ and 277.1951 in Met14 suggested that oxidation had occurred in the cyclopentane ring. The product ion spectra of Met13 exhibited addition of 15.9949 Da to the fragment ions of $\mathrm{m} / \mathrm{z}, 295.2050$ and 277.1951G and addition of 15.9949 Da followed by water loss to the fragment ion of $\mathrm{m} / \mathrm{z}$ 245.1900 , suggesting oxidation had occurred on the decahydrophenanthren ring containing the ethyl moiety. The product ion spectra of Met9 exhibited an intense daughter ion peak at $\mathrm{m} / \mathrm{z}, 313.2162$, corresponding to NG, which was produced by 176 neutral loss, which is characteristic of glucuronide conjugation of the $-\mathrm{OH}$ attached to the cyclopentane ring. The putative metabolic pathway of NGMN and NG is shown in Fig. 5.
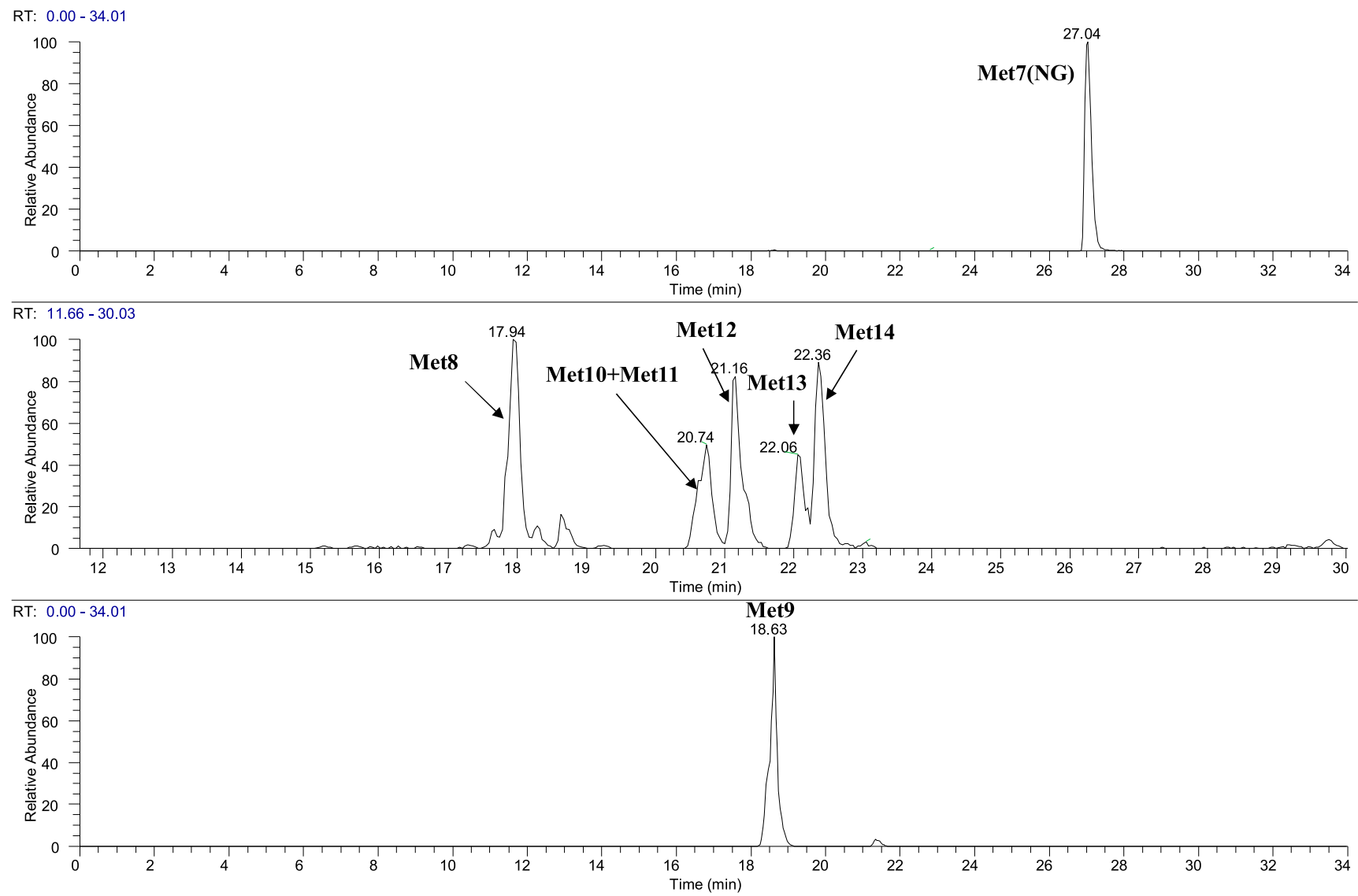

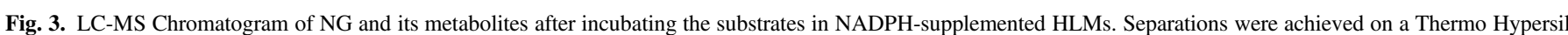
Gold C18 column and detected by a LTQ-Velos Orbitrap. The different panels contain the extracted ion chromatograms of parent and various metabolites. 
Metabolism of NGMN in the Presence of Recombinant P450s and HLMs. Initial screening of NGMN with recombinant P450 isoforms exhibited rapid metabolism of NGMN in the presence of rCYP3A4, whereas no significant turnover of parent was observed in the cases of other rCYPs (Fig. 6; Supplemental Fig. 1). Metabolite formation of NGMN was also analyzed in recombinant P450s (Fig. 7) and CYP2C9 and CYP2B6 showed the formation of Met1, Met3, and Met4. CYP3A5 did not form a significant amount of any metabolite. Hence, CYP3A4 was the predominant isoform involved in the metabolism of NGMN with minor contributions from CYP2B6 and CYP2C9. Interestingly, Met3, which was formed by CYP2B6 and CYP2C9 at 3 minutes, were further metabolized at 30 minutes, illustrating the importance of early time point measurements.

In HLMs, selective inhibitors KTZ (CYP3A4 and CYP3A5), CYP3cide (CYP3A4), and thio-TEPA (CYP2B6) substantially inhibited the disappearance of NGMN (Fig. 8A). Both KTZ and CYP3cide almost completely inhibited the disappearance of NGMN, suggesting that CYP3A4 played a predominant role in NGMN metabolism, consistent with results from the recombinant $\mathrm{P} 450$ s. Thio-TEPA also inhibited the metabolism of NGMN by around $50 \%$. Metabolite formation of NGMN also revealed the same trend (Fig. 8, B-F). At 30 minutes, Met1, Met3, Met4, Met5, and Met6 formations were inhibited in the presence of thioTEPA, whereas Met1, Met3, and Met4 were inhibited in the presence of sulphaphenazole (CYP2C9 inhibitor). Formation of all metabolites was inhibited by greater than $90 \%$ by KTZ and CYP3cide, consistent with parent disappearance data. Hence, based on the HLM data, CYP3A4 plays a major role in NGMN disappearance with minor contributions from CYP2B6 and CYP2C9. The $f_{\mathrm{m}}$ values for CYP3A4 and CYP2B6 were determined by eq. 1 , using the HLMs in the presence of inhibitor data, and were found to be 0.57 and 0.43 , respectively.

Metabolism of NG in the Presence of Recombinant P450s and HLMs. Turnover of NG was observed only in the presence of rCYP3A4 and rUGT1A1, whereas no turnover was observed in the cases of other recombinant P450s and recombinant UGTs (Supplemental Figs. 1 and 4). In HLMs, NG depletion was inhibited by $60 \%$ and $100 \%$ in the presence of KTZ and ATV. This translated to an $f_{\mathrm{m}}$ value of 0.6 for CYP3A4 and a combined $f_{\mathrm{m}}$ value of 1 for CYP3A 4 plus UGT1A1. In the presence of KTZ, Met2-7 formation was inhibited by more than $90 \%$, whereas Met1 formation was inhibited by $60 \%$.

DDI Prediction. Predicted fold-AUC changes for NGMN (as a victim) upon coadministration of LPR, RTV, ATV, and EFZ (perpetrators) were determined using the equations described previously, and the in vitro values shown in Table 4 . For both the ATV/RTV and LPR/RTV coadministered perpetrators, the fold NGMN AUC increase predicted was between 2.1- and 2.3-fold, while the observed fold change in NGMN AUC increase was around 1.8-fold. Hence, the predicted and actual DDI predictions were very close to each other. Irrespective of

TABLE 3

Metabolite profile of NG after incubation of $30 \mu \mathrm{M}$ NG in $1 \mathrm{mM}$ NADPHsupplemented $1 \mathrm{mg} / \mathrm{ml}$ HLMs

\begin{tabular}{lccc}
\hline Parent/Metabolite & $\mathrm{RT}$ & $\mathrm{MH}^{+}$ & Relative Abundance (UV) \\
\hline & & & $\%$ \\
NG & 27.04 & 313.2162 & 88.0 \\
Met8 & 17.94 & 329.2111 & 2.0 \\
Met9 & 18.63 & 489.2483 & 6.0 \\
Met10 & 20.74 & 329.2111 & 1.0 \\
Met11 & 20.74 & 329.2111 & 1.0 \\
Met12 & 21.16 & 329.2111 & 1.0 \\
Met13 & 22.05 & 329.2111 & 1.0 \\
Met14 & 22.36 & 329.2111 & 1.0 \\
\hline
\end{tabular}

$\mathrm{MH}^{+}$, Positive ion mass; RT, Retention Time. which inhibitor concentration was used-total $C_{\max }$, free $C_{\max }$, or portal vein concentration - the predicted fold AUC changes of NGMN were between 2.1- and 2.3-fold. When total perpetrator $C_{\max }$ and portal vein concentrations of the inhibitor were used, the NGMN AUC fold change was $\sim 2.3$-fold. For EFZ, the predicted fold decrease in the NGMN AUC was 0.35 (65\% reduction in the AUC) when total $C_{\max }$ was used and 0.70 when portal vein concentration was used. When free $C_{\max }$ was included in the equation only a $10 \%$ reduction in the AUC was predicted. The observed AUC fold reduction was 0.36 (64\% reduction in the AUC), very similar to the 0.35 -fold change predicted using total $C_{\max }$. All predicted and observed AUC changes are summarized in Table 4.

\section{Discussion}

Maintaining efficacious levels of the active OTC components is very important in women of child bearing age avoiding pregnancy. While EE (a component of OTC) metabolism is well studied and DDIs can be rationalized, norgestimate is more complex. Norgestimate by itself does not circulate but forms an active metabolite NGMN, and determining the altered exposures of NGMN is important. Until now, the metabolism and disposition of norgestimate had been studied in numerous studies, but the metabolism of NGMN was not investigated.

The metabolites of NGMN (Fig. 5) are hydroxylation on the decahydronaphthalene ring (Met1), di-hydroxylation on the ethyl, dodecahydro-1H-cyclopentanaphthalene moiety to produce (Met2), hydroxylation on ethyl decahydronaphthalene (Met3), hydroxylation on the ethyl moiety to produce (Met4), hydroxylation on the cyclopentane ring to form Met5, hydroxylation on decahydrophenanthren 2(3-H) oxime (Met6), and deoximation to produce NG (Met7). The metabolic pathway of NG (Fig. 5) includes oxidation on the ethyl and cyclopentane moiety to produce Met11 and Met14, respectively, oxidation on ethyldecahydronaphthalene (Met8, Met10, and Met12), hydroxylation on the dodecahydrophenanthrene ring (Met13), and direct glucuronidation on the $\mathrm{OH}$ group to produce Met9.

The reaction phenotyping results clearly indicate a dominant role for CYP3A4-mediated metabolism of NGMN $\left(f_{\mathrm{m}}\right.$ of 0.57$)$ with a lesser role for CYP2B6, even lesser role for CYP2C9, and no role for CYP3A5. The implication of CYP3A4 in the metabolism of NGMN is consistent with numerous clinical trials, wherein it was found that daclatasvir, alitretinoin, rosuvastatin, rifaximin, laropiprant, sofosbuvir, ledipasvir, dolutegravir, dexloxiglumide, tenofovir disoproxil fumarate (a prodrug of tenofovir), saxaglipitin, and raltegravir did not influence the exposures of NGMN (Simonson et al., 2004; Roy et al., 2005;

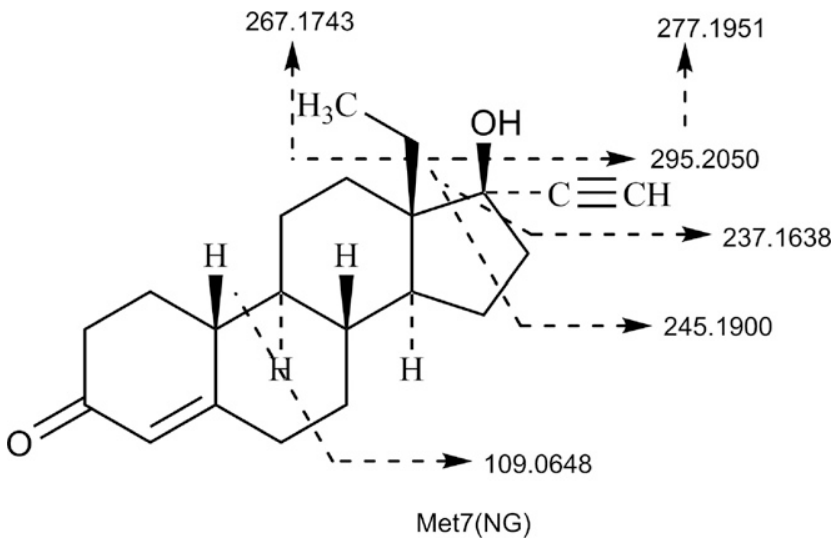

Fig. 4. Fragmentation of NG after its fragmentation in an LTQ-Velos-Orbitrap using collision-induced disassociation fragmentation. 


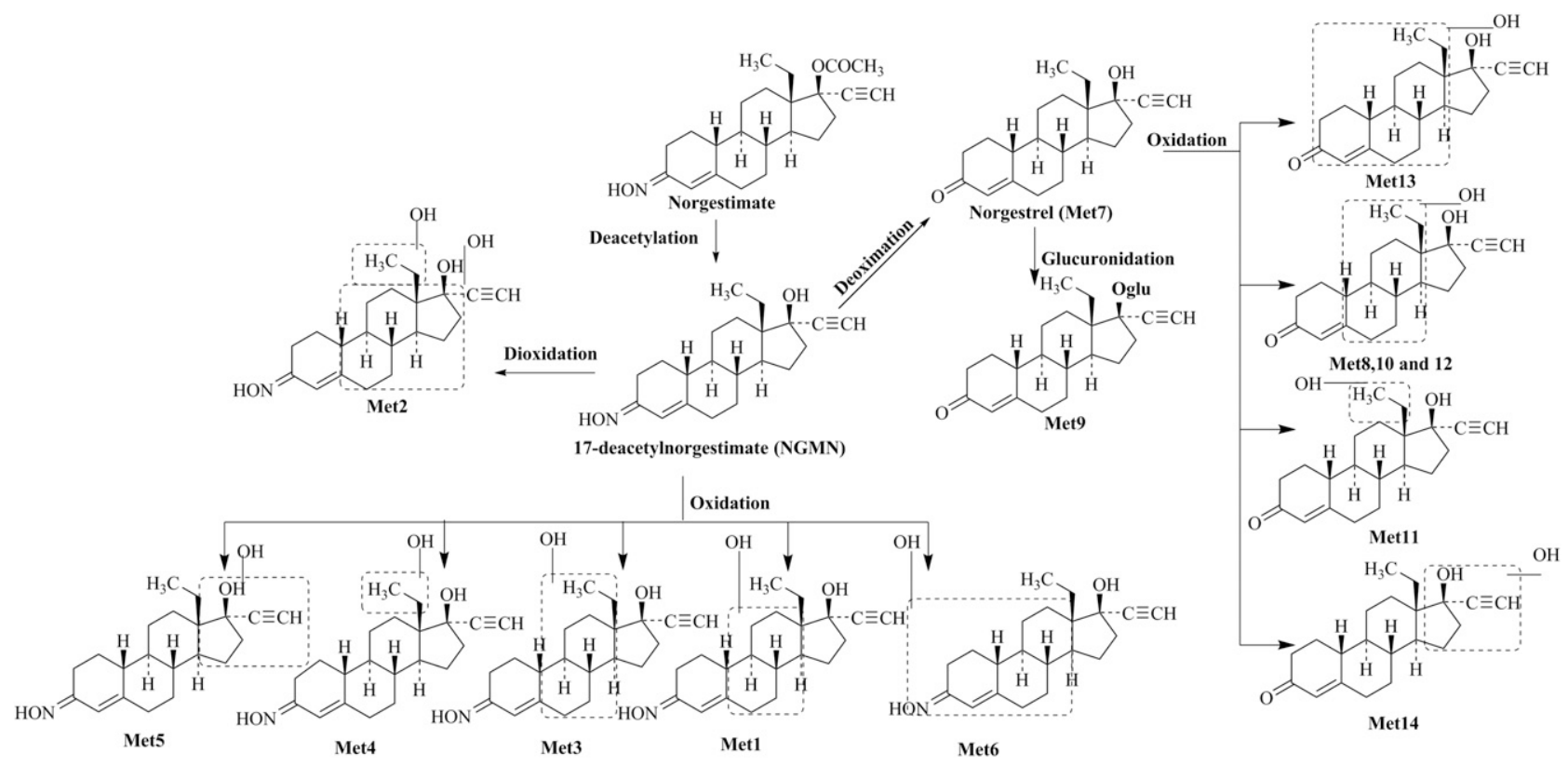

Fig. 5. Metabolic pathway of norgestimate and downstream putative metabolic pathway of NGMN and NG (Met7) in HLMs.

Kearney and Mathias, 2009; Schwartz et al., 2009; Anderson et al., 2011; Schmitt-Hoffmann et al., 2011; Upreti et al., 2012; Bifano et al., 2014; German et al., 2014; Song et al., 2015). The lack of an interaction is consistent with the data presented in this paper since none of these compounds are inducers/inhibitors of CYP3A4. In contrast, compounds that inhibit or induce CYP3A4 such as cobicistat, LPR/RTV and RTV/ ATV combinations, and EFZ significantly altered the exposure and pharmacokinetics of NGMN (Vogler et al., 2010; German, 2011; Sevinsky et al., 2011; Zhang et al., 2011). A fixed dose combination ofelvitegravir/cobicistat/emtricitabine/tenofovir disoproxil fumarate increased NGMN's exposure by greater than 2-fold, which is consistent with cobicistat's irreversible CYP3A4 inhibition properties (German, 2011). A dose of $300 \mathrm{mg} / 100 \mathrm{mg}$ of ATV/RTV caused $68 \%, 85 \%$, and $102 \%$ increases in $C_{\max }$, AUC, and $C_{24 \mathrm{~h}}$, respectively, for NGMN (Zhang et al., 2011). In another study, the patch NGMN AUC increased by $83 \%$ when LPR and RTV (400/100 mg) were coadministered with OTC (Vogler et al., 2010). In light of our data showing that NGMN is a substrate of CYP3A4, the increases in $C_{\max }$ are reasonable since LPR, RTV, and ATV are CYP3A4 inhibitors. Decreases of 46\%, 62\%, and $84 \%$ in NGMN $C_{\max }$, AUC, and $C_{24 \mathrm{~h}}$ were observed when $600 \mathrm{mg}$ (daily dosing) of EFZ was coadministered with OTC (14 days of codosing) (Sevinsky et al., 2011). No effect was observed on the EE pharmacokinetic parameters. EFZ is an inducer of CYP3A4 in vivo, which could explain the reason for NGMN exposures declining substantially (Siccardi et al., 2013). EFZ also decreased NG exposures by $60 \%-80 \%$, consistent with the fact that NG is metabolized by CYP3A4 and UGT1A1 (Sevinsky et al., 2011). It should be mentioned that in the ATV/RTV and LPR/RTV DDI studies, there was a reduction in EE exposure that was offset by an increases in NGMN exposure. Hence, in both of these studies (Zhang et al., 2011, Vogler et al., 2010), the authors did not anticipate a loss in contraceptive efficacy despite the observed DDIs. In contrast, when EFZ was coadministered there was no effect on the EE AUC but there were significantly reduced NGMN levels, which prompted the authors (Sevinsky et al., 2011) to recommend barrier contraception to overcome loss of efficacy.

The DDI predictions are summarized in Table 4 and show that in all of the inhibition DDI instances the observed and predicted DDIs were within 1.3-fold of each other. In the cases of LPR/RTV and ATV/RTV coadministration, when either free, total perpetrator $C_{\max }$, or free portal vein concentration was used there was very minimal impact on the NGMN AUC prediction even though the inhibitor concentrations were several fold apart. Since the perpetrators are potent irreversible and reversible inhibitors, even the free $C_{\max }$ is high enough to cause maximal inhibition. Hence, the only factor governing the NGMN DDI is $f_{\mathrm{m}}$, and the maximal DDI change is essentially $1 /\left(1-f_{\mathrm{m}}\right)$, which is 2.3 -fold and, in turn, is very close to the observed 1.8 -fold change. If the perpetrators are also CYP2B6 and CYP2C9 inhibitors, a higher AUC change can be expected. In the case of induction by EFZ, when the total $C_{\max }$ concentration was considered, the predicted AUC change was around 0.36 (64\% decline in the NGMN AUC), almost identical to the observed change of 0.35 . The predicted to actual AUC changes were larger when the free $C_{\max }$ and portal vein concentrations were used (1.9- to 2.5-fold difference, respectively). Therefore, in the case of inhibition and induction, the actual NGMN AUC changes are very close to the

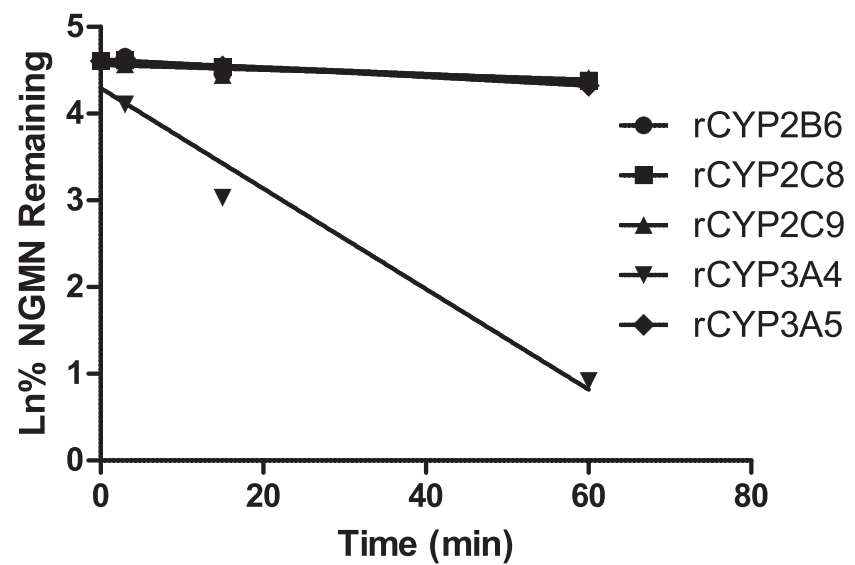

Fig. 6. The first-order disappearance of NGMN in the presence of various recombinant P450s. 

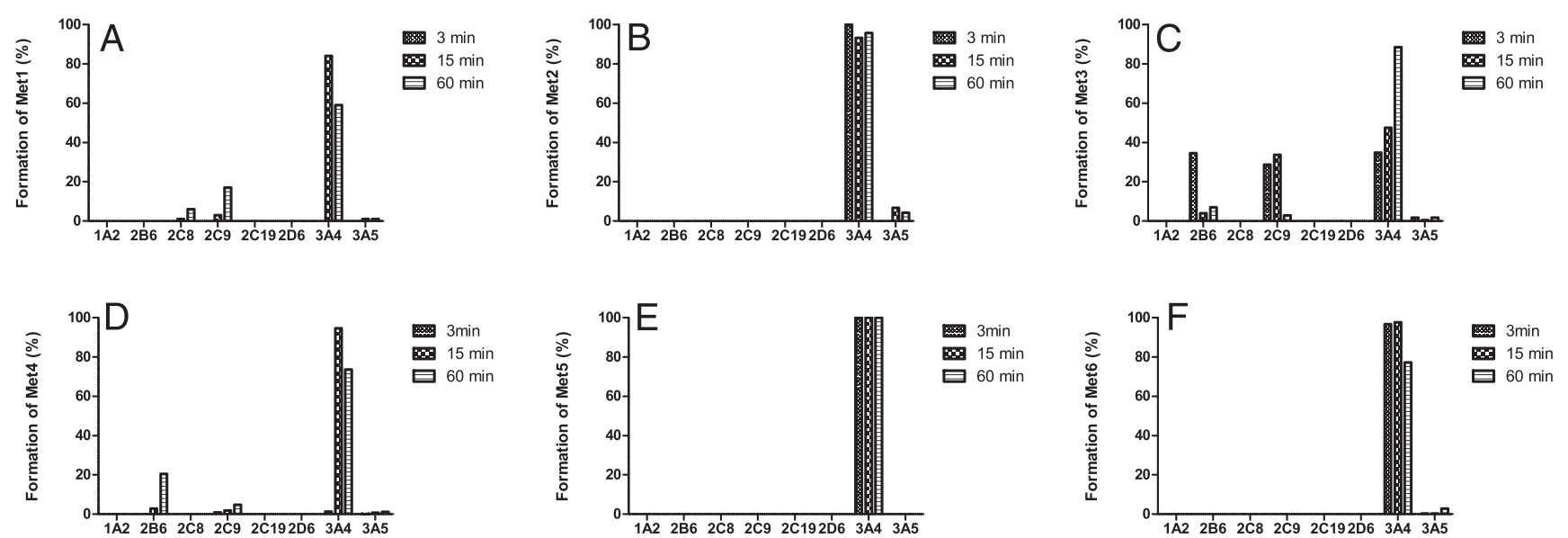

Fig. 7. Metabolite formation of NGMN in the presence of various recombinant P450s. Met1, Met2, Met3, Met4, Met5, and Met6 are shown in (A-F), respectively.

observed NGMN AUC changes when the total $C_{\max }$ values of the perpetrator are considered.

To determine which parameter had the greatest impact on the predicted fold NGMN AUC changes, a sensitivity analysis was performed wherein the values of $f_{\mathrm{m}}, K_{\mathrm{I}}, k_{\text {inact }}$, and inhibitor concentration were varied over a wide range (Fig. 9). From these analyses, it is clear that the NGMN $f_{\mathrm{m}}$ value has the most dramatic effect on the fold NGMN AUC change. The AUC change was from 1-fold to greater than 300 -fold as the $f_{\mathrm{m}}$ value ranged from 0 to 1 (Fig. 9A). In contrast, the $C_{\max }$ value had a minimal effect on the fold AUC (Fig. 9B). At very low perpetrator $C_{\max }$ values (close to $1 \mathrm{nM}$ ), the fold AUC change was $\sim 1$. From a $C_{\max }$ value of $100 \mathrm{nM}$ onward the fold AUC changed reached 2-fold and did not vary much thereafter, plateauing at a 2.3 -fold AUC change. Another important observation is that even though a combination of inhibitors was used (LPR/RTV or ATV/RTV), the fold AUC change was not additive, consistent with the fact that the $C_{\max }$ value had a minimal impact on the AUC change in the sensitivity analyses. The perpetrator $k_{\text {inact }}$ and $K_{\mathrm{I}}$ values also did not have a dramatic impact on the fold AUC change (Fig. 9, C and D).

DDIs involving protease inhibitors (PIs) are usually complex and paradoxical since they not only inhibit CYP3A4 but also induce CYP3A4 and CYP2B6. For example, at steady state, RTV does not change oral clearance of alprazolam but a single dose of RTV reduces the oral clearance of alprazolam (Liu et al., 2012). Additionally, upon chronic administration, RTV is capable of autoinduction despite CYP3A4 reversible and irreversible inhibition (Kirby et al., 2011). The $\mathrm{EC}_{50}$ and $E_{\max }$ values for RTV induction of $3 \mathrm{~A} 4$ have been incorporated into our DDI predictions; however, for LPR and ATV such data are not available. Additionally, while all three PIs have the potential to induce CYP3A4 and CYP2B6 at the transcript level, this induction may be masked by reversible and irreversible inhibition at the activity level, and hence accurate $E_{\max }$ and $\mathrm{EC}_{50}$ values are unavailable (Kharasch et al., 2008; Kirby et al., 2011; Liu et al., 2012). In our studies we predicted a 2.3-fold increase in the AUC based on the $f_{\mathrm{m}}$ value of 0.57; however, the observed AUC changes were around 1.8-fold. Induction of CYP3A4 and CYP2B6 could boost metabolism, compensating for the inhibition that leads to the lower AUC changes than predicted.

An accurate measure of $F_{\mathrm{g}}$ is essential to assess DDIs due to intestinal inhibition; however, such data are not available in the literature for the compounds under scrutiny. For low $F_{\mathrm{g}}$ compounds, much of the drug is in the intestine to exert its inhibitory effect and the extent of inhibition is high, and vice versa for compounds with a high $F_{\mathrm{g}}$ value. ATV and RTV have high oral bioavailability $(>60 \%)$ signifying a high $F_{\mathrm{g}}$ value (Zeldin
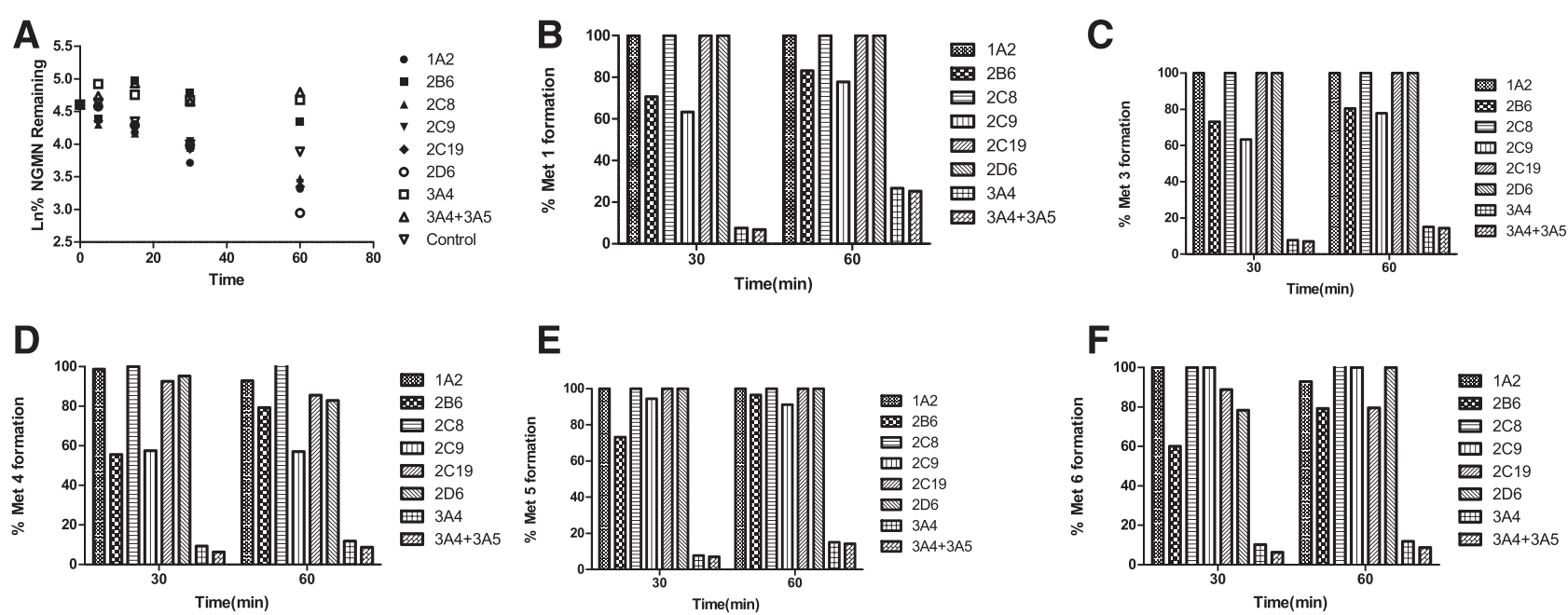

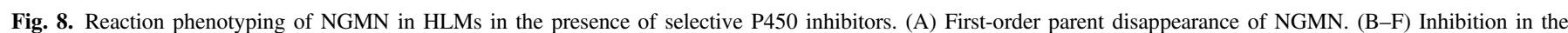
formation of Met1, Met3, Met4, Met5, and Met6, respectively. 
TABLE 4

Predicted and observed AUC changes of NGMN determined retrospectively from clinical trials

The perpetrator in vitro values shown in Table 3 were used for the DDI predictions. Equations 2-4, as defined in Materials and Methods, were used to calculate the fold changes in the AUC. LPR and RTV were coadministered in one study (Vogler et al., 2010), while ATV and RTV were coadministered in another study (Zhang et al., 2011). Hence, the fold AUC changes are the same for LPR and RTV (study 1) and ATV and RTV (study 2). EFZ was dosed in a separate study (Sevinsky et al., 2011).

\begin{tabular}{|c|c|c|c|c|c|}
\hline \multicolumn{3}{|c|}{ Perpetrator } & \multicolumn{2}{|c|}{ NGMN } & \multirow{2}{*}{ Predicted/Observed Ratio } \\
\hline Concentration Paradigm & Dose & Concentration & Predicted $\mathrm{AUC}_{\mathrm{i}} / \mathrm{AUC}$ & Observed $\mathrm{AUC}_{\mathrm{i}} / \mathrm{AUC}$ & \\
\hline & $m g$ & $n M$ & & & \\
\hline Total $\mathrm{C}_{\max }$ & LPR (400) & 14,790 & 2.3 & 1.82 & 1.28 \\
\hline Free $C_{\max }$ & LPR (400) & 118 & 2.2 & 1.82 & 1.24 \\
\hline$I_{\mathrm{h}}$ & LPR (400) & 42,526 & 2.3 & 1.82 & 1.28 \\
\hline Total $C_{\max }$ & RTV study 1 (100) & 745 & 2.3 & 1.82 & 1.28 \\
\hline Free $C_{\max }$ & RTV study 1 (100) & 2 & 2.1 & 1.82 & 1.17 \\
\hline$I_{\mathrm{h}}$ & RTV study 1 (100) & 9249 & 2.3 & 1.82 & 1.28 \\
\hline Total $C_{\max }$ & $\operatorname{ATV}(300)$ & 9616 & 2.3 & 1.83 & 1.27 \\
\hline Free $\mathrm{C}_{\max }$ & ATV (300) & 1298 & 2.3 & 1.83 & 1.26 \\
\hline $\mathrm{I}_{\mathrm{h}}$ & ATV (300) & 29,667 & 2.3 & 1.83 & 1.27 \\
\hline Total $\mathrm{C}_{\max }$ & RTV study 2 (100) & 2053 & 2.3 & 1.83 & 1.27 \\
\hline Free $C_{\max }$ & RTV study 2 (100) & 6 & 2.3 & 1.83 & 1.27 \\
\hline$I_{\mathrm{h}}$ & RTV study 2 (100) & 9253 & 2.3 & 1.83 & 1.27 \\
\hline Total $C_{\max }$ & EFZ (600) & 21,161 & 0.4 & 0.36 & 0.98 \\
\hline Free $C_{\max }$ & EFZ (600) & 127 & 0.9 & 0.36 & 2.49 \\
\hline$I_{\mathrm{h}}$ & EFZ (600) & 126,842 & 0.7 & 0.36 & 1.94 \\
\hline
\end{tabular}

and Petruschke, 2004; Colombo et al., 2006; Rathbun and Liedtke, 2011). Additionally, combination PIs have even greater $\% F$ than individually dosed PIs due to inhibition of metabolism as evidenced by the multifold increase in AUC values. Given the high $F_{\mathrm{g}}$ values likely for all PIs, the AUC change due to intestinal inhibition is likely to be low. Since accurate $F_{\mathrm{g}}$ values were not available, and the impact of intestinal inhibition was likely to be low, we have not included it in our analyses.

Norgestimate is a widely prescribed contraceptive agent and owes its efficacy to its active metabolite NGMN, whose levels are likely to be altered when enzymes involved in its metabolism are inhibited or induced. Any perturbations of NGMN levels will likely lead to adverse events or loss of efficacy. Hence, understanding and predicting NGMN DDIs is very important to safe implementation of a norgestimate dosing regimen. The data presented in this paper enable a prospective understanding of NGMN DDIs.

In summary, detailed biotransformation studies of both NGMN and NG in HLMs demonstrated that NGMN was metabolized to produce NG and several oxidative metabolites, whereas NG undergoes biotransformation to produce several oxidative metabolites and a glucuronide metabolite. The CYP3A $4 f_{\mathrm{m}}$ value of NGMN was determined to be 0.57 and the NGMN AUC changes observed in several clinical DDI studies were found to be within 1.3-fold of the predicted AUC changes.
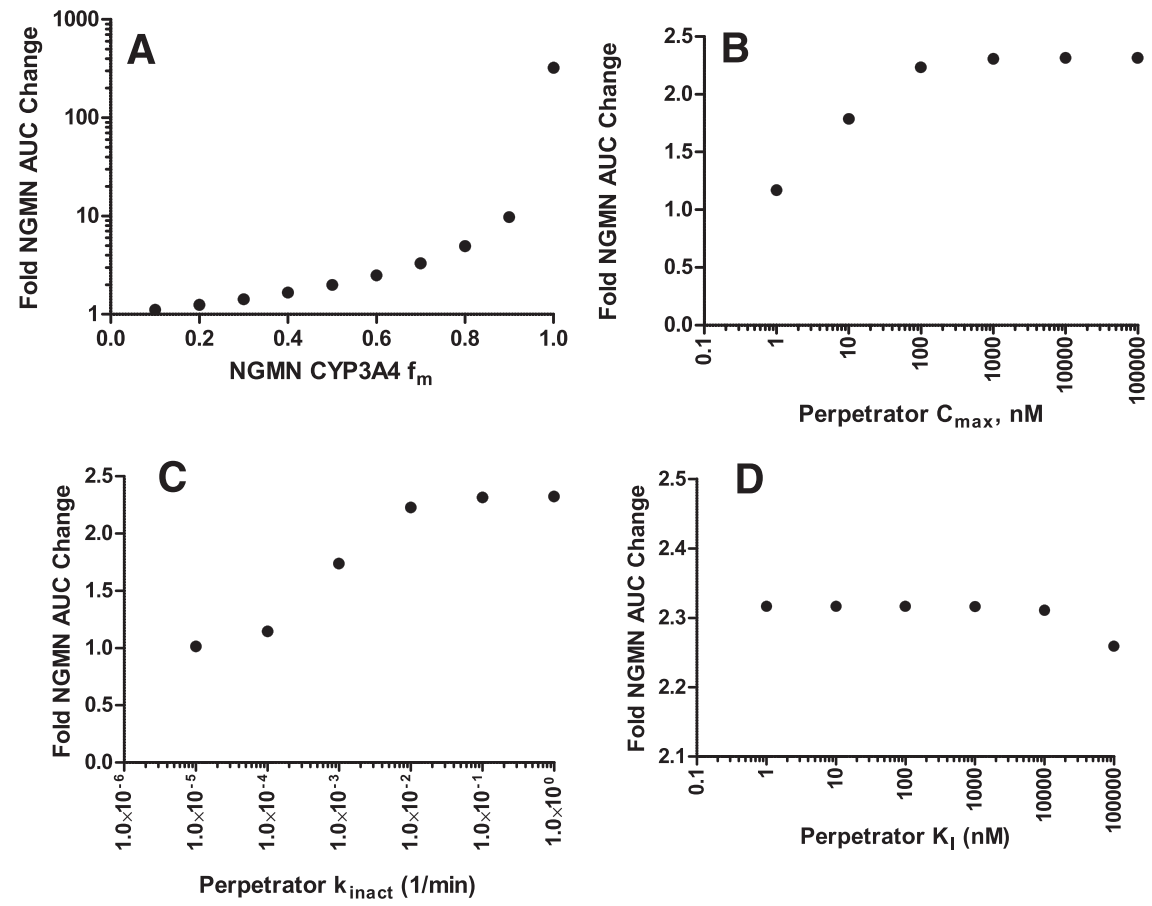

Fig. 9. Effect of various parameters on the NGMN foldAUC change for inhibition and induction-mediated DDIs of NGMN. While the AUC change is of NGMN (victim), the $C_{\max }, K_{\mathrm{I}}$, and $k_{\text {inact }}$ are the perpetrator properties as described in Table 1 . The values of $f_{\mathrm{m}}, k_{\text {inact }}, K_{\mathrm{I}}$, and $C_{\max }$ used are $0.57,0.11(1 / \mathrm{min}), 1000 \mathrm{nM}$, and $14.8 \mu \mathrm{M}$, respectively. (A-D) Effect of $f_{\mathrm{m}}, C_{\mathrm{max}}, k_{\text {inact }}$, and $K_{\mathrm{I}}$, respectively. 


\section{Authorship Contributions}

Participated in research design: Ahire, Sinha, Brock, Iyer, Mandlekar, Subramanian.

Conducted experiments: Ahire, Sinha.

Performed data analysis: Ahire, Sinha, Subramanian.

Wrote or contributed to the writing of the manuscript: Ahire, Sinha, Iyer, Mandlekar, Subramanian.

\section{References}

Almond LM, Hoggard PG, Edirisinghe D, Khoo SH, and Back DJ (2005) Intracellular and plasma pharmacokinetics of efavirenz in HIV-infected individuals. J Antimicrob Chemother 56: 738-744.

Alton KB, Hetyei NS, Shaw C, and Patrick JE (1984) Biotransformation of norgestimate in women. Contraception 29:19-29.

Anderson MS, Hanley WD, Moreau AR, Jin B, Bieberdorf FA, Kost JT, Wenning LA, Stone JA Wagner JA, and Iwamoto M (2011) Effect of raltegravir on estradiol and norgestimate plasma pharmacokinetics following oral contraceptive administration in healthy women. $\mathrm{Br} \mathrm{J} \mathrm{Clin}$ Pharmacol 71:616-620.

Aweeka FT, Stek A, Best BM, Hu C, Holland D, Hermes A, Burchett SK, Read J, Mirochnick M, and Capparelli EV; International Maternal Pediatric Adolescent AIDS Clinical Trials Group (IMPAACT) P1026s Protocol Team (2010) Lopinavir protein binding in HIV-1-infected pregnant women. HIV Med 11:232-238.

Becker H (1990) Supportive European data on a new oral contraceptive containing norgestimate. Acta Obstet Gynecol Scand Suppl 152:33-39.

Bifano M, Sevinsky H, Hwang C, Kandoussi H, Jiang H, Grasela D, and Bertz R (2014) Effect of the coadministration of daclatasvir on the pharmacokinetics of a combined oral contraceptive containing ethinyl estradiol and norgestimate. Antivir Ther 19:511-519.

Bringer J (1992) Norgestimate: a clinical overview of a new progestin. Am J Obstet Gynecol 166 1969-1977.

Colombo S, Buclin T, Franc C, Guignard N, Khonkarly M, Tarr PE, Rochat B, Biollaz J, Telenti A Decosterd LA, et al. (2006) Ritonavir-boosted atazanavir-lopinavir combination: a pharmacokinetic interaction study of total, unbound plasma and cellular exposures. Antivir Ther 11:53-62.

Corson SL (1990) Efficacy and clinical profile of a new oral contraceptive containing norgestimate. U.S. clinical trials. Acta Obstet Gynecol Scand Suppl 152:25-31.

Delille CA, Pruett ST, Marconi VC, Lennox JL, Armstrong WS, Arrendale RF, Sheth AN, Easley KA, Acosta EP, Vunnava A, et al. (2014) Effect of protein binding on unbound atazanavir and darunavir cerebrospinal fluid concentrations. J Clin Pharmacol 54:1063-1071.

Ernest CS, 2nd, Hall SD, and Jones DR (2005) Mechanism-based inactivation of CYP3A by HIV protease inhibitors. J Pharmacol Exp Ther 312:583-591.

Fahmi OA, Maurer TS, Kish M, Cardenas E, Boldt S, and Nettleton D (2008) A combined model for predicting CYP3A4 clinical net drug-drug interaction based on CYP3A4 inhibition, in activation, and induction determined in vitro. Drug Metab Dispos 36:1698-1708.

German P, Moorehead L, Pang P, Vimal M, and Mathias A (2014) Lack of a clinically important pharmacokinetic interaction between sofosbuvir or ledipasvir and hormonal oral contraceptives norgestimate/ethinyl estradiol in HCV-uninfected female subjects. J Clin Pharmacol 54:1290-1298.

German P, Wang M, Warren D, and Kearney B (2011). Pharmacokinetic interaction between norgestima/ethinyl estradiol and EVG/COBI/FTC/TDF single tablet regimen, in Proccedings of 12th International Workshop on Clinical Pharmacology of HIV Therapy; 2011 April 13-15, Miami, FL.

Hariparsad N, Nallani SC, Sane RS, Buckley DJ, Buckley AR, and Desai PB (2004) Induction of CYP3A4 by efavirenz in primary human hepatocytes: comparison with rifampin and phenobarbital. J Clin Pharmacol 44:1273-1281.

Huber J (1991) Clinical experience with a new norgestimate-containing oral contraceptive. Int $J$ Fertil 36 (Suppl 1):25-31.

Hyland R, Dickins M, Collins C, Jones H, and Jones B (2008) Maraviroc: in vitro assessment of drug-drug interaction potential. Br J Clin Pharmacol 66:498-507.

Kafrissen ME (1992) A norgestimate-containing oral contraceptive: review of clinical studies. Am J Obstet Gynecol 167:1196-1202.

Kalvass JC, Maurer TS, and Pollack GM (2007) Use of plasma and brain unbound fractions to assess the extent of brain distribution of 34 drugs: comparison of unbound concentration ratios to in vivo P-glycoprotein efflux ratios. Drug Metab Dispos 35:660-666.

Kaplan B (1995) Desogestrel, norgestimate, and gestodene: the newer progestins. Ann Pharmacother 29:736-742.

Kearney BP and Mathias A (2009) Lack of effect of tenofovir disoproxil fumarate on pharmacokinetics of hormonal contraceptives. Pharmacotherapy 29:924-929.

Kharasch ED, Mitchell D, Coles R, and Blanco R (2008) Rapid clinical induction of hepatic cytochrome P4502B6 activity by ritonavir. Antimicrob Agents Chemother 52:1663-1669.

Kirby BJ, Collier AC, Kharasch ED, Dixit V, Desai P, Whittington D, Thummel KE, and Unadkat JD (2011) Complex drug interactions of HIV protease inhibitors 2: in vivo induction and in vitro to in vivo correlation of induction of cytochrome P450 1A2, 2B6, and $2 \mathrm{C} 9$ by ritonavir or nelfinavir. Drug Metab Dispos 39:2329-2337.
Liu L, Mugundu GM, Kirby BJ, Samineni D, Desai PB, and Unadkat JD (2012) Quantification of human hepatocyte cytochrome P450 enzymes and transporters induced by HIV protease inhibitors using newly validated LC-MS/MS cocktail assays and RT-PCR. Biopharm Drug Dispos 33:207-217.

Madden S and Back DJ (1991) Metabolism of norgestimate by human gastrointestinal mucosa and liver microsomes in vitro. J Steroid Biochem Mol Biol 38:497-503.

McGuire JL, Phillips A, Hahn DW, Tolman EL, Flor S, and Kafrissen ME (1990) Pharmacologic and pharmacokinetic characteristics of norgestimate and its metabolites. Am J Obstet Gynecol 163:2127-2131.

Rathbun RC and Liedtke MD (2011) Antiretroviral drug interactions: overview of interactions involving new and investigational agents and the role of therapeutic drug monitoring for management. Pharmaceutics 3:745-781.

Roy P, Jakate AS, Patel A, Abramowitz W, Wangsa J, Persiani S, and Kapil R (2005) Effect of multiple-dose dexloxiglumide on the pharmacokinetics of oral contraceptives in healthy women. J Clin Pharmacol 45:329-336.

Schmitt-Hoffmann AH, Roos B, Sauer J, Schleimer M, Schoetzau A, Leese PT, Weidekamm E, and Maares J (2011) Influence of alitretinoin on the pharmacokinetics of the oral contraceptive ethinyl estradiol/norgestimate. Clin Exp Dermatol 36 (Suppl 2):4-11.

Schwartz JI, Liu F, Wang YH, Pramanik B, Johnson-Levonas AO, Gutierrez MJ, Lai E, and Wagner JA (2009) Effect of laropiprant, a PGD2 receptor 1 antagonist, on estradiol and norgestimate pharmacokinetics after oral contraceptive administration in women. Am J Ther 16: $487-495$.

Sevinsky H, Eley T, Persson A, Garner D, Yones C, Nettles R, Krantz K, Bertz R, and Zhang J (2011) The effect of efavirenz on the pharmacokinetics of an oral contraceptive containing ethinyl estradiol and norgestimate in healthy HIV-negative women. Antivir Ther 16:149-156.

Siccardi M, Olagunju A, Seden K, Ebrahimjee F, Rannard S, Back D, and Owen A (2013) Use of a physiologically-based pharmacokinetic model to simulate artemether dose adjustment for overcoming the drug-drug interaction with efavirenz. In Silico Pharmacol 1:4.

Simonson SG, Martin PD, Warwick MJ, Mitchell PD, and Schneck DW (2004) The effect of rosuvastatin on oestrogen \& progestin pharmacokinetics in healthy women taking an oral contraceptive. Br J Clin Pharmacol 57:279-286.

Song IH, Borland J, Chen S, Wajima T, Peppercorn AF, and Piscitelli SC (2015) Dolutegravir has no effect on the pharmacokinetics of oral contraceptives with norgestimate and ethinyl estradiol. Ann Pharmacother 49:784-789.

Upreti VV, Hsiang CB, Li L, Xu X, LaCreta FP, and Boulton DW (2012) Effect of saxagliptin on the pharmacokinetics of the active components of Ortho-Cyclen ${ }^{\circledR}$, a combined oral contraceptive containing ethinyl estradiol and norgestimate, in healthy women. Diabetes Obes Metab 14 $1155-1157$.

U.S. Department of Health and Human Services (2012) Drug Interaction Studies-Study Design, Data Analysis, Implications for Dosing, and Labeling Recommendations.

Vogler MA, Patterson K, Kamemoto L, Park JG, Watts H, Aweeka F, Klingman KL, and Cohn SE (2010) Contraceptive efficacy of oral and transdermal hormones when co-administered with protease inhibitors in HIV-1-infected women: pharmacokinetic results of ACTG trial A5188. J Acquir Immune Defic Syndr 55:473-482.

Weemhoff JL, von Moltke LL, Richert C, Hesse LM, Harmatz JS, and Greenblatt DJ (2003) Apparent mechanism-based inhibition of human CYP3A in-vitro by lopinavir. J Pharm Pharmacol 55:381-386.

Wild MJ, Rudland PS, and Back DJ (1991) Metabolism of the oral contraceptive steroids ethynylestradiol and norgestimate by normal (Huma 7) and malignant (MCF-7 and ZR-75-1) human breast cells in culture. J Steroid Biochem Mol Biol 39:535-543.

Wild MJ, Rudland PS, and Back DJ (1993) Metabolism of the oral contraceptive steroids ethynylestradiol, norgestimate and 3-ketodesogestrel by a human endometrial cancer cell line (HEC-1A) and endometrial tissue in vitro. J Steroid Biochem Mol Biol 45:407-420.

$\mathrm{Xu} C$ and Desta Z (2013) In vitro analysis and quantitative prediction of efavirenz inhibition of eight cytochrome P450 (CYP) enzymes: major effects on CYPs 2B6, 2C8, 2C9 and 2C19. Drug Metab Pharmacokinet 28:362-371.

Yang X, Atkinson K, and Di L (2016) Novel cytochrome P450 reaction phenotyping for lowclearance compounds using the hepatocyte relay method. Drug Metab Dispos 44:460-465.

Zeldin RK and Petruschke RA (2004) Pharmacological and therapeutic properties of ritonavirboosted protease inhibitor therapy in HIV-infected patients. J Antimicrob Chemother 53:4-9.

Zhang D, Chando TJ, Everett DW, Patten CJ, Dehal SS, and Humphreys WG (2005) In vitro inhibition of UDP glucuronosyltransferases by atazanavir and other HIV protease inhibitors and the relationship of this property to in vivo bilirubin glucuronidation. Drug Metab Dispos 33: 1729-1739.

Zhang J, Chung E, Yones C, Persson A, Mahnke L, Eley T, Xu X, and Bertz R (2011) The effect of atazanavir/ritonavir on the pharmacokinetics of an oral contraceptive containing ethinyl estradiol and norgestimate in healthy women. Antivir Ther 16:157-164.

Address correspondence to: Murali Subramanian, Biocon Bristol-Myers Squibb Research and Development Center, Syngene International Ltd., Biocon Park Plot 2 \& 3 Bommasandra IV Phase Bangalore-560 099, India. E-mail: murali. subramanian@syngeneintl.com 